\title{
WTO आणि ग्रामीण विकासात कृषी
}

\section{Agriculture in the WTO and Rural Development}

\section{Dr. Rakshit Madan Bagde,}

Assistant Professor, Late. Mansaramji Padole Arts College, Ganeshpur Bhandara ORCID iD - 0000-0002-7507-0244

Web of Science ResearcherID - AAF-2760-2020

SSRN - Author ID: 4770534

Authenticus ID - R-00J-YM2

Vidwan-ID : 221858

rakshitbagde@gmail.com

प्राचिन काळापासूनच शेती हा भारतीय लोकांचा प्रमूख व्यवसाय राहिलेला आहे. आज $65 \%$ लोकसंख्या शेतीवर विविध बाबतीत अवलंबून आहे. एके काळी भारतीय अर्थव्यवस्थेचा कणा समजली जाणारी कृषीचा कणा आज वाकत चाललेला आहे. याला मुख्य कारण म्हणजे सरकारी व्यवस्थेचे कृषीवरिल दुर्लक्ष आणि नव्याने सुरू झालेली जागतिकीकरणाची प्रकीया होय.

अजपावेतो ग्रामीण अर्थव्यवस्था टिकून आहे ती कृषी या एकाच घटकावर त्यातही आता जागतिकीकरणाच्या प्रकीयेची झड पोहचून ही अर्थव्यवस्था विस्कळी होण्याच्या वाटेवर आहे .

1 जानेवारी 1995 रोजी जागतिक व्यापार संघटनेच्या करारावर सहचा करून भारताने शेती क्षेत्राला जागतिकीकरणाच्या प्रकीयेत समावून घेतले. या बाबतीत 16 डिसेंबर 1999 रोजी भारत आणि अमेरिका दरम्यान गुप्त करार होवून भारताला आपल्या संरक्षित 715 कृषी वस्तूवरिल संख्यात्मक निर्बंध ही दुर करावे लागले. गॅट करार, डंकेल प्रस्ताव, विश्व व्यापार संघटना, आंतरराष्ट्रीय नाणेनिधी, जागतिक बँक या जागतिक संस्थांच्या माध्यमातून जागतिकीकरणाची संकल्पना साकार झालेली आहे .

\section{WTO च्या माध्यमातून शेतीच्या बाबतीत विविध अटी लादण्यात आल्या-}

1) शेतीला घरघुती अनुदान कमी करणे. 
2) शेतीला निर्यात अनुदान कमी करणे.

3) प्रशुल्क कमी करून घरघुती बाजार बंधन मुक्त करणे.

4) कृषी बियांचे पेटंट आणि व्यापारिक चिन्ह.

गॅटच्या उरुग्वे फेरीत ट्रिप्स नुसार पेटंट, कॉपी राईट, ट्रेडमार्क या बाबतीत नियम तयार केले असून ते बंधनकारक आहेत. ट्रिप्सचे नियम सजीवांना लागू होत असल्यामुळे जनूके, पेशी, बियाणे, वनस्पती व प्राणी यासंबंधी पे टंट प्रस्ताव करता येतो. WTO ने सदोष पेटंट पद्धती संपूर्ण जगभर लादली आहे

केली.

भारत सरकारने 28 जुलै 2000 रोजी राष्ट्रीय कृषी धोरणाची घोषणा त्यात -

1) कृषी विकासाला गती देण्याकरिता खाजगीकरणाचा वापर करणे .

2) ठेका कृषी तसेच पट्टचाने देण्याची व्यवस्था करणे.

3) कृषी क्षेत्रातील खाजगीकरणाला प्रोत्साहन देणे .

4) WTO च्या करारानुसार मात्रात्मक निर्बंध कमी केल्याने किंमतीतील उतार चढावापासून शेतकज्यांना संरक्षण देण्याकरिता निर्यातीवर भर देणे .

5) देशातील कृषी बाजारांना उदार (खाजगी)बनविणे.

6) व्यापारी शेतीवरील कर निर्धारणाचे समीक्षण करणे .

7) देशातील कृषी मालाच्या वाहतुकीतील प्रतिबंध कमी करणे .

8) उत्पादन शुल्काचे समीक्षण करणे.

जागतिक व्यापार संघटनेने अंमलबजावणि सूरू केलेल्या "स्ट्रक्चरल अडजेस्टमेंट प्रोग्रॉममनुसार" भारत सारकारने जे धोरण राबवीले ते ग्रामीण शेतीला घातक असे आहे .

सन 1920-21 मध्ये प्रती व्यक्ती कृषी योग्य क्षेत्र 1.11 एकर वरून आता फक्त 0.32 एकर प्रती व्यक्ती इतके कमी राहिले आहे तर गैर कृषी क्षेत्राचा हिस्सा 
196.6 लाखा हेक्टर वरून वाढून 244.8 हेक्टर झालेला आहे. याचा स्पष्ट प्रभाव एकूणच कृषी उत्पादनावर दिसू लागलेला आहे.

\section{कृषी क्षेत्राचे उत्पादन}

\begin{tabular}{|l|l|l|l|l|l|l|l|l|l|l|}
\hline योजना & \multicolumn{2}{|c|}{$\begin{array}{c}\text { तीळ } \\
\text { लाख टन }\end{array}$} & \multicolumn{2}{|c|}{$\begin{array}{c}\text { उस } \\
\text { लाख टन }\end{array}$} & \multicolumn{2}{|c|}{$\begin{array}{c}\text { कापूस } \\
180 \\
\text { कि. लाख } \\
\text { गट्ठे }\end{array}$} & $\begin{array}{r}\text { ताग } \\
170 \text { कि. लाख } \\
\text { गट्ठे }\end{array}$ & \multicolumn{2}{|c|}{$\begin{array}{l}\text { खाद्यान्न } \\
\text { लाख टन }\end{array}$} \\
\hline & लक्ष्य & वास्तविक & लक्ष्य & वास्तविक & लक्ष्य & वास्तविक & लक्ष्य & वास्तविक & लक्ष्य & वास्तविक \\
\hline 8 वी. & 230 & 250 & 2750 & 2770 & 140 & 143 & 95 & 110 & 2100 & 1990 \\
\hline 9 वी. & 300 & 207 & 3360 & 3000 & 157 & 101 & -- & 116 & 2340 & 2110 \\
\hline
\end{tabular}

8 व्या आणि 9 व्या योजनेत अपेक्ष्कीत उत्पादनापेक्षा कमी उत्पादन झालेले तर कापूस,तीळ यांच्या उत्पादनात घट झालेली आपणास निदर्शनास येते .

भारतीय प्रती हेक्टर उत्पादनाचे विदेशी प्रती हेक्टर उत्पादनाशी तूलना करतातांदूळ - कि.ग्रा.प्रती हे .

गहू - कि.ग्रा. प्रती हे.

मिस्र 8769 टर्की 7076

यु.एस.ए. 7205

फान्स 6632

जापान 6659

मिस्र 6357

भारत 2964

भारत 2742

वरिल दोन मुख्य पिकांची उत्पादकता पाहता भारतीय शेती अजून प्राथमीक अवस्थेत असल्याचे दिसून येते .

खाद्य सूरक्षेचा विचार करता 1990-91 ते 2005-06 या काळात प्रती व्यक्ती खाद्यान्यउपलब्धता 510 ग्राम वरून कमी होवून 422 ग्राम प्रती दिवस कमी झाले. विविध प्रकीरच्या डाळी या ग्रामीण जनतेचे आरोग्य टिकविण्यासाठी आवश्यक 
असणारे प्रोटीन पूरविण्याचे महत्वाचे माध्यम आहे. सन 1950-51 मध्ये प्रती व्यक्ती उपलब्धता 61 ग्राम प्रतीदिवस वरून सन 1990-91 मध्ये 42 ग्राम इतकी कमी झाली असे असूनही “भारतीय कृषी विज्ञान आणि संशोधन परिषदेने" यापूढे डाळीच्या नव्या जाती शोधण्याची आवश्यकता नसल्याचे म्हटले आहे. याचा परिणाम म्हणून डाळीची निर्यात वाढून कॅनडाला त्याचा सर्वाधीक फायदा होतो आहे.

निर्यात व्यापाराच्या बाबतीत कृषीचा विचार केल्यास 2003-04 मध्ये 11. $9 \%$ ने घसरून 2004-05 मध्ये $10.2 \%$ झाले. ही घसरण कायम राहून ऑक्टोबर 2005 पर्यंत $9.6 \%$ एवढी झाली. कृषी आयात 2003-04 मध्ये 3708.2 मी.अ. डॉलर होती. ती वाढून 2004-05 मध्ये 3811 मी.अ. डॉलर एवढी झाली.

ग्रामीण शेतकज्याला तीन कारणास्तव मोठचा प्रमाणात कर्जाची आवश्यकता भासते. 1. उत्पादन कार्यासाठी 2. आदाने खरेदी करिता 3 . मजुरी देण्याकरिता. कृषीचे व्यापारीकरण करण्यात आल्याने कर्जाची मागणीही वाढलेली आहे. NSS च्या सर्वेक्षणानुसार $43 \%$ शेतकज्यांनी सावकारी कर्ज काढले होते ज्यात $53 \%$ कर्ज $15 \%$ दराने तर $16 \%$ कर्ज $30 \%$ इतक्या मोठचा दराने काढले होते.

सन 2002 मध्ये एकूण कर्जाच्या $42.9 \%$ कर्ज सावकारी स्वसूपाचे होते ज्यामध्ये $27 \%$ ग्रामीण परिवार ऋणग्रस्त होते. यातच होणारी नापीकी, पूर आणि अवर्षण, नैसर्गीक प्रकोप, कमी कीमत इ. कारणास्तव ऋणाची परतफेड होवू न शकल्याने आणि सरकारचा उदासिनपणा यातून शेतकरी मोठचा प्रमाणात आत्महत्या करतो आहे .

भारतीय शेतकच्यांच्या आत्महत्या.

\begin{tabular}{|c|c|c|}
\hline सन & $\begin{array}{c}\text { शेतकज्यांच्या आत्महत्या } \\
\text { (अनुमानित) }\end{array}$ & आत्महत्याची टक्केवारी \\
\hline 1995 & 10,719 & 12.0 \\
\hline 1996 & 13,730 & 15.6 \\
\hline
\end{tabular}




\begin{tabular}{|l|l|l|}
\hline 1997 & 13,617 & 14.2 \\
\hline 1998 & 16,010 & 15.3 \\
\hline 1999 & 16,079 & 14.5 \\
\hline 2002 & 25,071 & 16.3 \\
\hline
\end{tabular}

सन 1995 ते 2002 पर्यंतची शेतकज्यांच्या आत्महत्येची आकडेवारी 15000 पर्यंत वाढलेली दिसून येते. कृषी प्रधान भारत देशाला याहून दुसरी अशोभनिय घटना इतर कोणतीही नसावी, शेतकरी मेल्यावर लाखो रूपये मदत करणारी आमची सरकार शेतकज्याला जगण्याकरिता 10-15 हजार रूपयाचीही मदत करीत नाही. या अशा सरकारी धोरणामूळेच मनुष्य वधाचा प्रकार वाढतच चाललेला आहे .

W.T.O. च्या रिपोर्टनुसार कृषी उत्पादन, उपभोग व विक्रीच्या प्रत्येक क्षेत्रात सरकारी हस्तक्षेपामुले जास्त अयोग्यता व उत्पादन नफा कमी झालेला आहे. शेवटी रिपोर्टमध्ये दावा करण्यात आला आहे की, उदारीकरणात सगळचांचा फायदा होईल. शेती क्षेत्रात सुधारणा घडविणाज्या नरसिंहम समितीने सार्वजनिक बँकांना कृषी क्षेत्रात दिल्या जाणाय्या कर्जात मोठचा प्रमाणात कपात करण्याची सूचना दिली व सल्ला दिला की $40 \%$ ऐवजी $10 \%$ कर्ज शेती क्षेत्रासाठी द्यावे

मुक्त आर्थिक धोरणाच्या अंमलबजावणीमुळे ग्रामीण भागातील जनतेत पोटापुरते अन्र विकत घेऊन ते खाण्याची क्रयशक्ती उरलेली नाही. रोजगार नाही तर पैसे नाही आणि पैसे नाही तर अत्र नाही म्हणून भूक भागविता येत नाही. सरकारने यातील रोजगारच कमी करून भुखबळीला पोषक वातावरण निर्माण केले आहे. सबसिडी कपात आणि अन्रधान्य भाववाढ यांच्यामुळे वाढलेले दारिद्रच हे मुख्यतः ग्रामीण भागात आहे . ब्रेटनवुड्डस संस्थांनी कर्ज घेणाज्या देशांमध्ये अन्रधान्य सबसिडी ही विशिष्ट वर्गाकरिता सिमीत करण्यावर भर दिला आहे. त्यामुळे गरिबांना स्वस्त धान्य दुकानातून अत्र मिळणे कठीण झाले आहे .

आजही भारतात 3 कोटी कुटूंब असे आहेत की ते शेत नांगरणीसाठी बैल-रेडा नसेल तर बैल-मनुष्य अशी जोडी करून शेत नांगरतात. परिणामी 
जागतिकीकरण अशा शेतकज्यांना काहीच देत नाही उलटपक्षी त्याला उरलेल्या शेतीतून विस्थापीत करण्यात येत आहे .

1993-94 पासून देशात बेरोजगारीचे प्रमाण वाढत चालले आहे. ग्रामीण भागात पुरुषांच्या बेरोजगारीचे प्रमाण 1993 - 94 मध्ये $5.6 \%$ वरून 2004 मध्ये $9.0 \%$ तर महिला बेरोजगारीचा विचार केल्यास ग्रामीण भागात 1993-94 मध्ये $5.6 \%$ वरून 2004 पर्यंत $9.3 \%$ पर्यंत वाढले आहे. ग्रामीण रोजगारीत एका अंदाजानुसार एकूण कृषी मजदूरांच्या $80 \%$ मजदूर हे अनुसूचित जातीचे आहेत. यातही मिळणारी मजूरी ही महाराष्ट्राचा विचार करता 2006-07 दरम्यान 45 रू. इतही होती.

जागतिकीकरणाच्या प्रकीयेत भारतात खाजगी आणि सरकारी अशी दोन स्वतंत्र क्षेत्र निर्माण झालेली आहेत. या खाजगी क्षेत्रात $15 \%$ पगारवाढ दरवर्षी होते आहे त्यात IIM मधून नुकतेच पास झालेल्यांना 365 दिवसांकरिता 5,000 रू प्रतीदिवस वेतन दिले जाते परंतू सरकारच्या राष्ट्रीय रोजगार गारंटी योजनेत फक्त 100 रू प्रतीदिवस मीळतात ते ही 100 दिवस कामाच्या अटीवर. वेतनाची ऐवढी मोठी तफावत समाजात अराजकता आणि असंतोष निर्माण करणारी आहे .

या बाबतीत माजी राष्ट्रपती के.आर.नारायणन इशारा देतात की, "खूप काळापासून सतावलेल्या धैर्यवान लोकात असंतोष निर्माण होवू शकतो जर खाजगीकरण, उदारीकरण आणि जागतिकीकीकरणाच्या त्रिआयामी मार्गात भारताच्या कमजोर वर्गाकरिता पैदल मार्ग निर्माण केला नाही." याचाच उलट परिणाम म्हणजे आज देशात 200 जिल्हयात नक्षलवाद जोर पकडतो आहे .

आता पाण्यावर आधारीत अर्थव्यवस्था निर्माण होणाज्याची चिन्हे दिसत आहेत. पाण्याच्या बँका (Water Bank) स्थापन होतील. पाणी सम्राट (Water Lord) तयार होतील. आता श्रीमंत राष्ट्रे वॉटर बँका स्थापण्याच्या तयारीला 
लागलेली आहेत. पाण्याचे खाजगीकरण झाले तर पाण्याचे दर मोठचा प्रमाणात वाढून कालव्याचे पाणी शेतकज्यांना परवडणार नाही. परिणामी धान्य, डाळी, तेलबियांचे उत्पादन घटून गरीब जनतेला अन्नधान्य परवडेनासे होऊन उपासमारीच्या संकटाने देश वेढला जाईल. बाबासाहेबांच्या काळात एकाच तळयाचा प्रश्न होता आता गावोगावच्या तळयांसाठी संर्घण करावा लागणार आहे .

एप्रिल 2000 मध्ये सेझ पॉलिसीचा प्रस्ताव भारतात ठेवण्यात आला. महाराष्ट्रात सेझ अधिनियम 2003 मध्ये लागू करण्यात आला आणि 2005 मध्ये सेझ बिल पास करण्यात आले भारतात सिंगूर आणि नंदीग्राम हा सेझ आणि मुक्त अर्थव्यवस्थेचा परिणाम आहे. सेझ करिता जमीन अधिग्रहण करतेवेळी शहरांना आणि बाजूच्या खोडयांना लक्ष केल्या जात आहे. परंतू लवकरच त्याची झळ ग्रामीण कृषीवर पडण्याची शक्यता नाकारता येणार नाही. मृणाल गोरे आणि न्या. प. बा. सावंत यांनी केलेल्या अभ्यासानुसार बृहन्मुबंईच्या जमीन व्यवहाराची माहिती आश्चर्यकारक आहे -

1) सात मोठचा जमीनधारकांकडे 12,926 एकड जमीन आहे.

2) 338 मोठचा जमीनधारकांकडे 17,097 एकड जमीन आहे .

मोठचा जमीनधारकांची 30,023 एकड जमीन असेल तर एकूण जमीनीत सर्वसामान्यांचा वाटा किती असेल ते समजूनच येते .

सिताराम येचुरी यांनी दिलेल्या माहितीनुसार महाराष्ट्र सरकारने 50,000 रु. प्रती हेक्टर ने शेतकच्यांकडून जमीन विकत घेऊन ती जमीन दर हेक्टरी 62 लाखाला एका कंपनीला विकली. पुढे यांचा कंपनीने ती जमीन 'सेझ' साठी 10 कोटी प्रती हेक्टर भावाने विकली. यावरून सरकार शेतकज्यांचा किती हितचिंतक आहे ते कळते . 
चीन देशाच्या सेझचे सत्य दर्शन 'विल द बोट सिंग द वाटर्स ?' या पुस्तकातून दिसून येते. तेथील शेतकरी सेझमुळे कसे भुकेकंगाल झाले आहेत याची सत्य परिस्थिती त्या ग्रंथात आहे. त्यामुळे चीन सरकारने त्या ग्रंथावर बंदी घातली आहे. आज सेझ खाली संपूर्ण भारतात $5,50,000$ एकड जमीन अधिग्रहित करण्यात येत आहे. त्यामुळे शेतकज्यांवर निश्चितच भीख मागण्याची पाळी येणार आहे. एवढचा मोठचा प्रमाणात सुपीक जमीन सेझ व्याप्त झाली तर कृषीवर अवलंबून असणाय्या असंख्य लोकांचे काय होईल?

आज भारत एक धोकादायक मार्गातून जातो आहे एकीकडे भारत चमकतो आहे,यात विनिर्माण आणि सेवा क्षेत्राचा अधिक विकास होतो आहे याउलट शेती क्षेत्रात घोर अंधकार आणि विषमता वाढत चालली आहे. दुसय्या हरितकांतीच्या जलद सूरूवातीची आवश्यकता भासत चालली आहे. ज्यात शहरी आणि ग्रामीण संपत्तीची विषमता कमी करणे आवश्यक आहे. अन्यथा प्रो.पी.आर.ब्रम्हानंद म्हणतात त्या प्रमाणे, "आम्हाला देशीय हिताला प्रथम स्थान दचायला हवे आणि याकरिता देशातील शेतकच्यांच्या हितांकरिता आम्हाला जागतिक व्यापार संघटनेला सोडावे लागले तरी, मी याला अनुचित समजत नाही."

संदर्भ ग्रंथ -

1 एस.के.मिश्र आणि वी.के.पूरी - भारती अर्थव्यवस्था, हिमालया प्रकाशन, नागपूर 2003

2 रूद्र दत्त आणि सुंदरम - भारतीय अर्थव्यवस्था, एस. चंद एन्ड कंपनी ली. रामनगर, दिल्ली 2009

3 संपादक - साहित्य भवन प्रतियोगिता सीरीज,भारतीय अर्थव्यवस्था एक दृष्टि में साहित्य भवन पब्लिशर्स एण्ड डिस्ट्रीब्यूटर्स, आगरा, 2006.

4 अडमिरल विष्णू भागवत - जागतिकीकरण नविन गुलामगिरी, समता प्रकाशन, नागपूर 2006.

5 उत्तम कांबळे - जागतिकीकरण आणि दलितांचे प्रश्न, सुगावा प्रकाशन पुणे, 2002.

6 रक्षित मदन बागडे - मुक्त आर्थिक धोरणामूळे निर्माण होणाय्या समस्यांचे डॉ. आंबेडकरांच्या आर्थिक तत्वज्ञानातून चिकित्सक विश्लेषण, एम. ए. डॉ. आंबेडकर विचारधारा विभाग लघुशोध प्रबंध, 2008.

7 पुरूशोत्तम लांडगे - डॉ. आंबेडकरांचा राज्य समाजवाद आणि जागतिकीकरण : एक चिकित्सक अध्ययन, लघुशोध प्रबंध, डॉ. आंबेडकर विचारधारा, 2001-02

8 चित्रलेखा कौसल - सेझ, शून्यदीप प्रकाशन, नागपूर 2007 